\title{
Clinical evidence of photobiomodulation therapy (PBMT) on implant stability and success: a systematic review and meta- analysis
}

Yuan Chen, Caojie Liu, Xinlei Chen and Anchun Mo*i]

\begin{abstract}
Background: Photobiomodulation therapy (PBMT), a type of light therapy that uses the concept of photobiomodulation, is developed to promote bone healing. Clinical studies have been conducted to assess the influence of PBMT on dental implant stability and success rate. This is the first systematic review and meta-analysis to assess the effect of PBMT and methodological quality of these studies on implants in human clinical trials.

Methods: An electronic search was performed in Pubmed, Embase, and the Cochrane Controlled Register of Trials (CENTRAL).

Results: Initially, 675 articles were identified, among which only 8 met the inclusion criteria. Four of the 8 studies presented a low risk of bias, whereas the other 4 were of moderate risk. Our review focused on implant success rates and implant stability measured at days 0 and 10, and at 3, 4, 6, and 12 weeks. No significant differences were observed between the PBMT group and the control group regarding implant stability or success rate.

Conclusions: The existing clinical studies did not provide sufficient evidence to observe positive effects of PBMT on implants in patients. An increased number of high-quality clinical randomized controlled trials (RCTs) are required to verify the data and to draw convincing conclusions.
\end{abstract}

Keywords: Dental implant, Implant stability, Implant success rate, Low level laser therapy, Photobiomodulation therapy

\section{Background}

Because of its excellent aesthetics, functional characteristics, and high success rates, implantology has been increasingly popular among patients with dentition defects [1]. However, there is still a risk of failure due to a number of complex factors, with lack of osseointegration as the main reason [2].

Osseointegration is a core definition in implantology, and describes the direct structural and functional connection between live bone and the surface of an implant [3]. Throughout osseointegration, osteoblasts and osteoclasts

\footnotetext{
* Correspondence: moanchun@163.com

Yuan Chen and Caojie Liu are contributed equally to this article and should be regarded as co-first authors.

Department of Oral Implantology, West China Hospital of Stomatology, Sichuan University, Chengdu 610041, China
}

interact and influence each other. Osseointegration is considered one of the most important determinants of implant stability [4], and is a key factor that determines success of the implant. However, a number of factors can influence osseointegration [5]. Therefore, increased attention has been paid to the physical, chemical, and biological attempts to promote osseointegration, one of which is photobiomodulation therapy (PBMT) [6].

PBMT, also known as low level therapy (LLLT), is defined as 'a type of noninvasive and nonthermal therapy based on non-ionizing light sources, including lasers, light-emitting diodes (LEDs), and broadband light, in the visible and infrared spectrum' [7]. In the 1990s, the Food and Drug Administration (FDA) approved laser therapy for oral treatment, therefore its application in surgery and endodontic treatment has been among the most

(c) The Author(s). 2019 Open Access This article is distributed under the terms of the Creative Commons Attribution 4.0 International License (http://creativecommons.org/licenses/by/4.0/), which permits unrestricted use, distribution, and 
popular topical treatments, e.g. treatment of mucosal leukoplakia, pediatric dental diseases, and alveolar osteitis [8]. Laser classification is complex and involves a large variety in different categories, such as excitation source, wavelength, and intensity. In medical applications, the power of the laser is an important parameter for therapy [9]. LED is an alternative for the laser because their effects on tissue are similar, and LED was given FDA approval [10].

In previous studies, the clinical effect of PBMT has been well characterized, e.g. it alleviates inflammation and pain, and promotes wound healing [11]. The bio-stimulating effect of PBMT has aroused the interest for research although there a clear mechanism of action is lacking [12]. Many studies have shown that PBMT contributes to bone healing $[13,14]$, and close attention has been given to the bio-stimulating effects of PBMT on osteoblast proliferation and osteogenesis after implant therapy [15]. Moreover, in previous studies it has been attempted to establish the most appropriate wavelength, dose, frequency, etc. to provide a protocol for the use of PBMT in implantology [16].

To ascertain the effect of PBMT on improving implant stability, previous studies have focused on animals, including rodents, rabbits, beagles, and primates. [2]. A number of studies and systematic reviews have been conducted, and suggested that PBMT provided a positive effect in animal models $[1,2,17]$. However, due to the lack of clinical data, these studies could not provide powerful evidence for a positive effect of PBMT in humans. Fortunately, a number of clinical trials have recently been published, thereby increasing the cohort of treated patients. Thus, in this systematic review and meta-analysis, we aimed to evaluate the clinical effects of PBMT on implant stability and success in humans.

\section{Methods}

This systematic review and meta-analysis was performed according to the Preferred Reporting Items for Systematic Reviews and Meta-Analyses (PRISMA) guidelines [18].

\section{Focused question}

It has been previously been established that PBMT promotes the osseointegration process in animals, therefore, our focused question was addressed based on the Participants, Interventions, Control and Outcomes (PICO) principle: 'For patients receiving implant treatment, does PBMT enhance implant stability and success rate?'

\section{Search strategy}

In this study, a literature search of the databases Pubmed, Embase, and the Cochrane Controlled Register of Trials (CENTRAL) was conducted up to November
2018. For each database Appropriate search algorithms were developed using the following terms: (laser OR laser therapy OR laser irradiation OR phototherapy OR low-level laser OR low-intensity laser OR low-output laser OR soft laser) AND (implant) AND (stability OR osseointegration). The search was limited to human subjects and without restriction in publication language.

Two blinded, independent investigators screened the titles and abstracts identified by the electronic search to select potentially eligible studies. Subsequently, the full text of the candidate studies was further evaluated to identify studies that met all inclusion criteria. To avoid missing any eligible studies, the references of all included articles and relevant reviews were also searched. In addition, a manual search was performed using the following dental implant and laser journals: Lasers in Medical Science; The International Journal of Oral and Maxillofacial Implants; The Journal of the American Dental Association; Journal of Oral and Maxillofacial Surgery; Journal of Periodontology; Photomedicine \& Laser Surgery, and The Journal of Prosthetic Dentistry. Agreement between reviewers in the selection procedure was calculated by the Cohen's kappa statistics, assuming $\kappa=0.6$ as an eligible score [19]. Any discrepancies were resolved by discussion or consultation with a third reviewer.

\section{Inclusion and exclusion criteria}

This review only focused on randomized controlled trials (RCTs) or quasi-RCTs reporting the effects of PBMT on implant stability and/or implant success rate in human clinical trials. Case reports, review papers, letters to the editor, monographs, in vitro studies, animal studies, and studies recruiting patients with systemic diseases or those being medically treated were excluded.

\section{Data collection and analysis}

The following information was extracted from the studies included in this review by two independent reviewers: first author (year), country, sample size, mean age (range), study type, the position of implant, type of implant and surface, bone condition, analysis performed, evaluation time, type of PBMT, wavelength, mode, output/energy (density), total dose per point (implant), exposure time, and frequency of laser treatment. The corresponding author was contacted to obtain any incomplete or missing data. Disagreements were resolved by discussion, with arbitration by a third reviewer, if necessary.

Statistical analysis was performed using RevMan 5.3 software [20] provided by the Cochrane Collaboration. For implant stability, the mean differences (MD), and 95\% confidence intervals (CI) were calculated. Implant success rate was classified as dichotomous data, thus the 
effect of intervention was estimated as a risk ratio (RR) with a $95 \% \mathrm{CI}$. The $\mathrm{I}^{2}$ statistic was used to evaluate trial heterogeneity using $\alpha=0.10$. A random-effects model was employed to analyze data exhibiting substantial heterogeneity $\left(\mathrm{I}^{2}>50 \%\right)$. In other cases, a fixed-effects model was used. Statistical significance was set at $\alpha<$ 0.05 (two-tailed $\mathrm{z}$ tests). If a meta-analysis could not be performed, data were summarized qualitatively.

\section{Quality assessment}

The quality of all the studies included in the review was assessed by two investigators who were blinded, using the Cochrane risk of bias assessment tool [21]. The agreement between reviewers was assessed based on Cohen's kappa statistics, assuming $\mathrm{\kappa}=0.6$ to be an eligible score [19]. Any disagreement was resolved by discussion, and a third reviewer was consulted if arbitration was required. The assessment of all articles encompassed seven domains: random sequence generation, allocation concealment, blinding of participants and outcome assessors, incomplete outcome data, selective reporting, and other bias. Each domain was divided into low risk of bias, unclear risk of bias, or high risk of bias. A study was regarded low risk only if all domains were evaluated as low risk. If one or more domains were evaluated as unclear, the study was categorized as being of moderate risk. Any domain examined as high risk resulted in that study being classified as high risk of bias.

\section{Results}

\section{Study characteristics}

During the search process, a total of 975 articles were identified (Fig. 1). After screening the titles and abstracts, the full texts of 14 articles were obtained, and further evaluated by two independent investigators (inter-reviewer agreement, kappa $=0.92$ ). Finally, 8

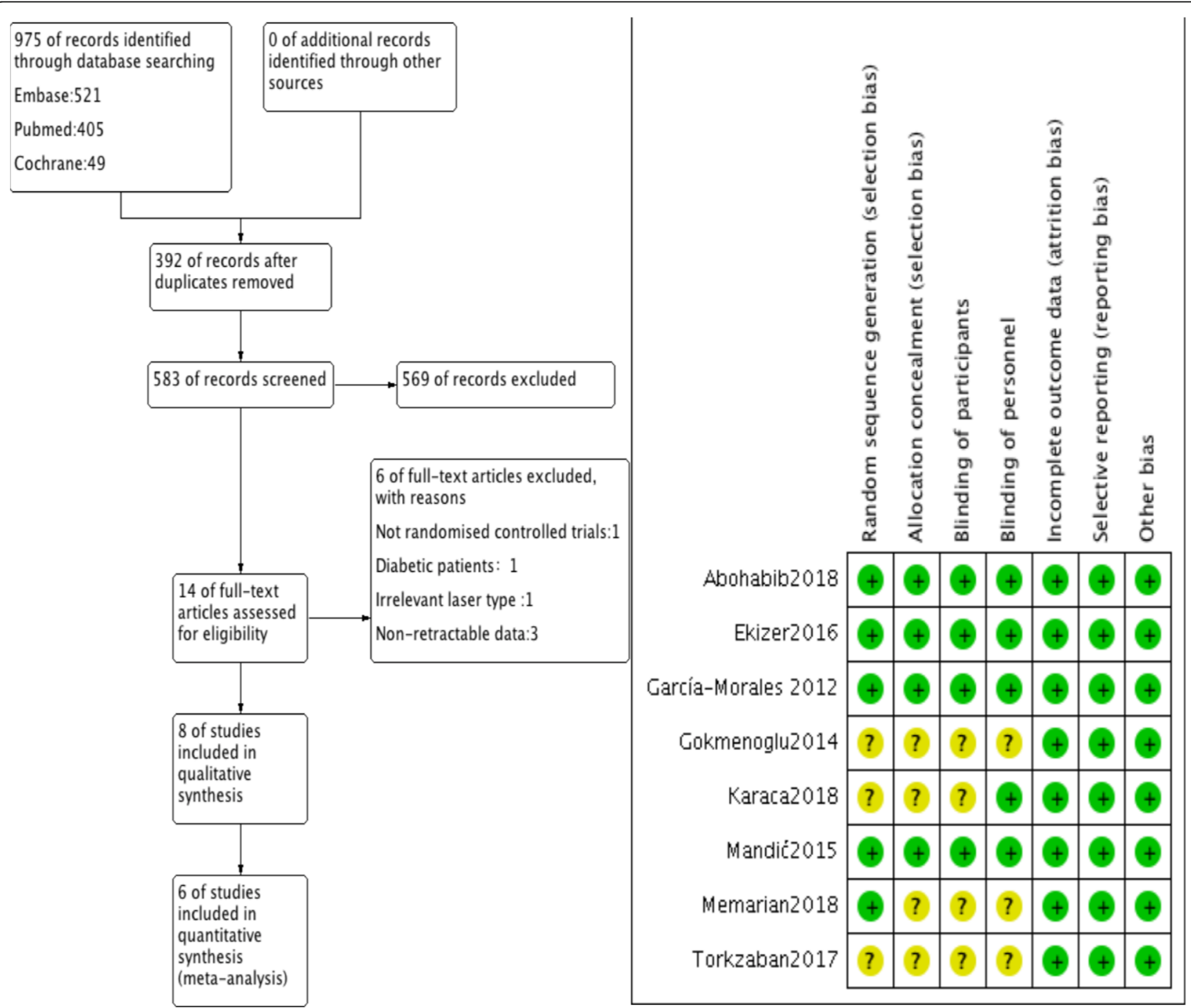

Fig. 1 Flowchart depicting the study selection process and risk of bias summary 
studies were selected, of which 6 were chosen for meta-analysis [22-29].

Six out of the 8 studies were published after 2015. Of these, 3 studies were conducted in Turkey, 2 in Iran, and the remaining 3 were conducted in Egypt, Serbia, and Brazil. In 7 studies, the mean age or age range was described, ranging from 13.1 to 75 years old. The timing of examinations varied among studies, and involved mostly 0 and 10 days, and 3, 4, 6, 12 weeks after dental implant insertion. The parameters of PBMT also varied among studies. Five used low level lasers with a continuous wave, 2 used LED, and 1 used a low level laser or LED. The wavelength of the PBMT varied from $618 \mathrm{~nm}$ to $940 \mathrm{~nm}$ with power output ranging from $20 \mathrm{~mW}$ to $1700 \mathrm{~mW}$. Further characteristics of the studies are summarized in Table 1, the parameters of the lasers are presented in Table 2.

\section{Quality analysis}

The risk of bias in the studies included in the review was assessed according to the Cochrane Handbook (Fig. 1). Four studies presented low risk of bias [24, 26, 27, $29]$, whereas the other 4 were of moderate risk [22, 23, 25, 28]. Unclear information about 'random sequence generation', 'blinding of participants', 'blinding of personnel' and 'allocation concealment' accounted for the risk factors. Of the 4 studies with moderate risk, the blinding to participants or allocation concealment was not mentioned. Furthermore, in 3 studies, blinding of personnel was not mentioned, and in 3 studies, the specific randomization methods were not mentioned. The agreement between the reviewers was 0.91 .

\section{Primary outcomes-the effect on implant stability}

Eight studies reported the effects of PBMT on implant stability at different follow-up times. Of these, the evaluation periods varied considerably. In our review, we only pooled the outcomes measured at days 0 and 10, and at 3, 4, 6, 12 weeks after implant placement. For data with implant stability quotient (ISQ) measurements, we conducted a meta-analysis, otherwise, the outcomes were only qualitatively described.

\section{Outcomes measured immediately and 10 days after implants were placed}

All 8 studies included in the review assessed implant stability immediately after the implant was placed. Among them, 6 studies used ISQ measurements, thereby enabling synthesis of the data by meta-analysis [22, 23, 2629 ]. The results of the meta-analysis verified that no significant differences were observed in implant stability between the PBMT group and the control group $\left(\mathrm{I}^{2}=\right.$ $23 \% ; P=0.63 ; \mathrm{MD}=0.28 ; 95 \% \mathrm{CI}$ : $-0.86-1.42$ ) (Fig. 2). One study reported resonance frequency analysis (RFA) values in Hertz and 1 reported Periotest values (PTV), and neither of showed significant differences between the 2 groups measured immediately after implantation $[24,25]$. Two of the 8 studies reported implant stability using the ISQ index measured 10 days after implant insertion. The results also demonstrated no significant differences between treatment and control groups $\left(\mathrm{I}^{2}=0 \%\right.$; $P=0.12 ; \mathrm{MD}=1.77 ; 95 \% \mathrm{CI}$ : $0.44-3.97$ ) (Fig. 2).

\section{Outcomes measured 3 and 4 weeks after placement of implants}

In five studies, implant stability data was provided and was measured 3 weeks after implant placement [23-25, 27, 29]. However, 1 reported RFA in Hertz and 1 reported PTV. Therefore, these 2 studies were not included in the meta-analysis [24, 25]. Although PBMT improved the ISQ index when compared with controls, a mean difference of 0.9 was not statistically significant $\left(\mathrm{I}^{2}=0 \% ; P=0.32 ; \mathrm{MD}=0.9 ; 95 \% \mathrm{CI}\right.$ : -0.88-2.67) (Fig. 3). Five studies reported outcomes 4 weeks after implant insertion [24-28], however those reporting PTV and RFA were excluded from the meta-analysis. No significant differences were observed in the results of the remaining 3 studies $(P=0.16)$, with very small heterogeneity in the data $\left(\mathrm{I}^{2}=0 \%, P=0.68\right)$ (Fig. 3). However, Abohabib et al. measured implant stability using RFA in Hertz and reported significant differences between the PBMT and control groups at 3 and 4 weeks after implant insertion (3 weeks: $P=0.032$; 4 weeks: $P=0.047$ ) [24]. Memarian et al. compared low level laser and LED with a control group [25], and the results indicated significant improvement after LED and low level laser treatment 3 and 4 weeks after implantation.

\section{Outcomes measured 6 weeks after implant placement}

In 4 studies, data concerning implant stability were measured 6 weeks after implant insertion [23, 24, 27, 29]. Abohabib et al. reported RFA in Hertz, demonstrating a significant difference between PBMT and the control group $(P=0.016)[24]$. In the remaining 3 studies, ISQ measurements were used and were thus included in the meta-analysis. PBMT provided no significant improvement in implant stability $\left(\mathrm{I}^{2}=0 \% ; P=0.71 ; \mathrm{MD}=0.32\right.$; 95\% CI: -1.35-1.98) (Fig. 4).

\section{Outcomes measured 12 weeks after implant placement}

In 4 studies, adequate implant stability data measured 12 weeks after placing of the implants were reported [23, 26, $28,29]$, on which meta-analysis was conducted. No significant differences were observed between PBMT groups and control groups, with significant heterogeneity in the data $\left(\mathrm{I}^{2}=73 \% ; P=0.50 ; \mathrm{MD}=1.02 ; 95 \% \mathrm{CI}:-1.97-4.01\right)$ (Fig. 4). 
Table 1 Characteristics of included studies

\begin{tabular}{|c|c|c|c|c|c|c|c|c|c|}
\hline Author(year) & Country & $\begin{array}{l}\text { Sample } \\
\text { size }\end{array}$ & $\begin{array}{l}\text { Mean age } \\
\text { (range) }\end{array}$ & $\begin{array}{l}\text { Study } \\
\text { type }\end{array}$ & $\begin{array}{l}\text { The position of } \\
\text { implant }\end{array}$ & $\begin{array}{l}\text { Type of implant and } \\
\text { surface }\end{array}$ & $\begin{array}{l}\text { Bone } \\
\text { condition }\end{array}$ & $\begin{array}{l}\text { Performed } \\
\text { analysis }\end{array}$ & Evaluation time \\
\hline Abohabib(2018) & Egypt & 15 & $20.9 \pm 3.4$ & $\begin{array}{l}\text { Split } \\
\text { mouth }\end{array}$ & $\begin{array}{l}\text { Between the } \\
\text { maxillary second } \\
\text { premolar and } \\
\text { first molar } \\
\text { buccally }\end{array}$ & $\begin{array}{l}\text { AbsoAnchor orthodontic } \\
\text { mini-implants (AbsoAnchor, } \\
\text { Dentos, Daegu, Korea) with } \\
\text { a diameter of } 1.5 \mathrm{~mm} \text { and } \\
\text { a length of } 8 \mathrm{~mm}\end{array}$ & $N R$ & $\begin{array}{l}\text { RFA in } \\
\text { Hertz }\end{array}$ & $\begin{array}{l}\text { Immediately } \\
\text { and 1,2,3,4, 6, } \\
8,10 \text { weeks } \\
\text { later }\end{array}$ \\
\hline Mandić(2015) & Serbia & 12 & $\begin{array}{l}61.28(55- \\
75)\end{array}$ & $\begin{array}{l}\text { Split } \\
\text { mouth }\end{array}$ & $\begin{array}{l}\text { Premolar and/or } \\
\text { molar maxillary } \\
\text { regions }\end{array}$ & $\begin{array}{l}\text { Self-tapping BlueSky } \\
\text { implants(Bredent, Germany) } \\
\text { with a diameter of } 4 \mathrm{~mm} \\
\text { and a length of } 10 \mathrm{~mm}\end{array}$ & $\begin{array}{l}\text { Type D3 and } \\
\text { D4 }\end{array}$ & $\begin{array}{l}\text { RFA in } \\
\text { ISQ }\end{array}$ & $\begin{array}{l}\text { Immediately } \\
\text { and } 1,2,3,4,5 \text {, } \\
6 \text { weeks later }\end{array}$ \\
\hline Karaca(2018) & Turkey & 25 & $\begin{array}{l}51.2 \pm \\
2.3(36- \\
64)\end{array}$ & NR & $\begin{array}{l}\text { Posterior } \\
\text { mandible }\end{array}$ & $\begin{array}{l}\text { DTI Implant } \\
\text { Systems(Istanbul, Turkey) } \\
\text { with a diameter of } 4 \text { or } 4.5 \\
\mathrm{~mm} \text { and a length of } 10 \\
\mathrm{~mm}\end{array}$ & $\begin{array}{l}\text { Minimum } 12 \\
\text { mm above } \\
\text { the } \\
\text { mandibular } \\
\text { canal and } \\
\text { minimum } 5.0 \\
\text { mm in width }\end{array}$ & $\begin{array}{l}\text { RFA in } \\
\text { ISQ }\end{array}$ & $\begin{array}{l}\text { Immediately } \\
\text { and } 6 \text { months } \\
\text { later }\end{array}$ \\
\hline $\begin{array}{l}\text { García-Morales } \\
\text { (2012) }\end{array}$ & Brazil & 8 & $36(20-55)$ & $\begin{array}{l}\text { Split } \\
\text { mouth }\end{array}$ & $\begin{array}{l}\text { Posterior } \\
\text { mandible }\end{array}$ & $\begin{array}{l}\text { XiVE-S implants(Dentsply } \\
\text { Friadent, Mannheim, } \\
\text { Germany) with a diameter } \\
\text { of } 3.8 \mathrm{~mm} \text { and a length of } \\
11 \mathrm{~mm}\end{array}$ & Type 2 & $\begin{array}{l}\text { RFA in } \\
\text { ISQ }\end{array}$ & $\begin{array}{l}\text { Immediately, } 10 \\
\text { days and } \\
3,6,9,12 \text { weeks } \\
\text { later }\end{array}$ \\
\hline Memarian(2018) & Iran & 12 & $N R$ & $\begin{array}{l}\text { Split } \\
\text { mouth }\end{array}$ & $\begin{array}{l}\text { Mandible(one in } \\
\text { the midline and } \\
\text { the other two at } \\
\text { the left and } \\
\text { right canine } \\
\text { teeth positions) }\end{array}$ & $\begin{array}{l}\text { DIO implants (Korea) with } \\
\text { resorbable blast media } \\
\text { surface(invasive fungal } \\
\text { infections-tissue level) }\end{array}$ & Type 2 or 3 & PTV & $\begin{array}{l}\text { Immediately } \\
\text { and } 3,4,8 \text { weeks } \\
\text { later }\end{array}$ \\
\hline Ekizer(2016) & Turkey & 20 & $\begin{array}{l}16.77 \pm \\
1.41 \\
(13.1-19)\end{array}$ & $\begin{array}{l}\text { Split } \\
\text { mouth }\end{array}$ & $\begin{array}{l}\text { Between the } \\
\text { roots of } \\
\text { maxillary first } \\
\text { molars and } \\
\text { second } \\
\text { premolars }\end{array}$ & $\begin{array}{l}\text { Screw-shaped titanium } \\
\text { orthodontic miniscrews with } \\
\text { a diameter of } 1.6 \mathrm{~mm} \text { and a } \\
\text { length of } 8 \mathrm{~mm}\end{array}$ & $N R$ & $\begin{array}{l}\text { RFA in } \\
\text { ISQ }\end{array}$ & $\begin{array}{l}\text { Immediately } \\
\text { and } 1,2,3 \\
\text { months later }\end{array}$ \\
\hline $\begin{array}{l}\text { Gokmenoglu } \\
\text { (2014) }\end{array}$ & Turkey & 15 & $\begin{array}{l}\text { LED: } 50.43 \\
\pm 9.25 \\
C: 45.87 \pm \\
13.46\end{array}$ & $N R$ & NR & $\begin{array}{l}\text { XiVE implants(Dentsply- } \\
\text { Friadent, Mannheim, } \\
\text { Germany) with a diameter of } \\
3.8(3.5-4.5) \mathrm{mm} \text { and a } \\
\text { length of } 11.0(11-11) \mathrm{mm} \text { in } \\
\text { LED group, while with a } \\
\text { diameter of } 4.5((3.8-4.5) \mathrm{mm} \\
\text { and a length of } 11.0 \text { (10.6- } \\
\text { 11.0)mm in control group }\end{array}$ & Type 2 or 3 & $\begin{array}{l}\text { RFA in } \\
\text { ISQ }\end{array}$ & $\begin{array}{l}\text { Immediately } \\
\text { and } 2,4,8,12 \\
\text { weeks later }\end{array}$ \\
\hline Torkzaban(2017) & Iran & 19 & $\begin{array}{l}\text { Female:43 } \\
\text { Male:40.8 }\end{array}$ & $N R$ & Maxillary teeth & $\begin{array}{l}\text { Dio implants (Dio UF, } \\
\text { Busan, Korea) with } \\
\text { a diameter of } 4 \text { or } \\
4.5 \mathrm{~mm} \text { a length } \\
\text { of } 10 \text { or } 11.5 \mathrm{~mm}\end{array}$ & $\begin{array}{l}\text { Type D3 and } \\
\text { D4 }\end{array}$ & $\begin{array}{l}\text { RFA in } \\
\text { ISQ }\end{array}$ & $\begin{array}{l}\text { Immediately,10 } \\
\text { days and } \\
3,6,12 \text { weeks } \\
\text { later }\end{array}$ \\
\hline
\end{tabular}

NR Not reported, RFA Resonant frequency analysis, PTV Periotest value, ISQ Implant stability quotient, C Control group

\section{Secondary outcomes-implant success rate}

Four studies reported the success rate of dental implants $[22,24,27,29]$. In a study by Karaca et al., only the overall success rate of all groups 6 months after implant insertion was reported (92\%) [22]. Thus, a meta-analysis was conducted on the remaining 3 studies. A forest plot revealed that the implant success rate of the PBMT group was similar to that of the control group $\left(\mathrm{I}^{2}=0 \%\right.$; $P=1 ; \mathrm{MD}=1 ; 95 \% \mathrm{CI}: 0.9-1.11$ ) (Fig. 5).

\section{Discussion}

Quality of the studies

Based on the quality assessment of the study, half of the included studies were considered to have moderate risk of bias. Among them, 2 studies presented unclear information about 'random sequence generation', 'allocation concealment,' 'blinding of participants', and the 'blinding of personnel', 1 study was vague about 'random sequence generation', 'allocation concealment', and 'blinding of 
Table 2 Parameters of PBMT in the included studies

\begin{tabular}{|c|c|c|c|c|c|c|c|}
\hline Author(year) & Type of LLLT & Wavelength & mode & $\begin{array}{l}\text { Output/ } \\
\text { energy(density) }\end{array}$ & $\begin{array}{l}\text { Total dose } \\
\text { Per } \\
\text { point(implant) }\end{array}$ & $\begin{array}{l}\text { Exposure } \\
\text { time }\end{array}$ & $\begin{array}{l}\text { Frequency of } \\
\text { laser treatment }\end{array}$ \\
\hline Abohabib(2018) & Biolase diode laser (Epic 10 Console) & $940 \mathrm{~nm}$ & $\begin{array}{l}\text { Continuous } \\
\text { wave }\end{array}$ & $1.7 \mathrm{w} ; 36 \mathrm{~J} / \mathrm{cm} 2$ & $102 \mathrm{~J} /$ implant & $60 \mathrm{~s}$ & $\begin{array}{l}\text { Immediately after } \\
\text { implant insertion } \\
\text { and } 7,14,21 \text { days } \\
\text { later }\end{array}$ \\
\hline Mandić(2015) & $\begin{array}{l}\text { GaAlAs laser(Medicolaser 637, } \\
\text { Technoline, Belgrade, Serbia) }\end{array}$ & $637 \mathrm{~nm}$ & $\begin{array}{l}\text { Continuous } \\
\text { wave }\end{array}$ & $\begin{array}{l}40 \mathrm{mw} ; 6.26 \mathrm{~J} / \\
\mathrm{cm} 2\end{array}$ & NR & NR & $\begin{array}{l}\text { Immediately after } \\
\text { implant insertion } \\
\text { and } 1,2,3,4,5,6,7 \\
\text { days later }\end{array}$ \\
\hline Karaca(2018) & $\begin{array}{l}\text { GaAlAs laser(Laser BTL-4000, Brno, } \\
\text { Czech Republic) }\end{array}$ & $830 \mathrm{~nm}$ & $\begin{array}{l}\text { Continuous } \\
\text { wave }\end{array}$ & $\begin{array}{l}86 \pm 2 \\
\mathrm{~mW} ; 92.1 \mathrm{~J} / \mathrm{cm} 2\end{array}$ & $\begin{array}{l}0.25 \mathrm{~J} / \text { point; } \\
5 \mathrm{~J} / \text { per teeth }\end{array}$ & $60 \mathrm{~s}$ & $\begin{array}{l}\text { Repeated every } \\
\text { two days for } 2 \\
\text { weeks }\end{array}$ \\
\hline $\begin{array}{l}\text { García- } \\
\text { Morales(2012) }\end{array}$ & $\begin{array}{l}\text { GaAlAs laser(Thera Lase, DMC, São } \\
\text { Carlos - SP, Brazil) }\end{array}$ & $830 \mathrm{~nm}$ & $\begin{array}{l}\text { Continuous } \\
\text { wave }\end{array}$ & $\begin{array}{l}86 \pm 2 \\
\mathrm{~mW} ; 92.1 \mathrm{~J} / \mathrm{cm} 2\end{array}$ & $\begin{array}{l}0.25 \mathrm{~J} / \text { point; } \\
5 \mathrm{~J} / \text { per } \\
\text { implant }\end{array}$ & $60 \mathrm{~s}$ & $\begin{array}{l}\text { Repeated every } \\
\text { two days for } 2 \\
\text { weeks }\end{array}$ \\
\hline Memarian(2018) & $\begin{array}{l}\text { Diode laser(Diode laser doctor smile } \\
810 \mathrm{~nm} \text {, Italy); LED(Osseopulse }{ }^{\mathrm{TM}} \mathrm{AR} \\
\text { 300, Biolux Research Ltd., Vancouver, } \\
\text { British Columbia, Canada) }\end{array}$ & $\begin{array}{l}\text { Laser:810 } \\
\text { nm } \\
\text { LED:626 nm }\end{array}$ & $\begin{array}{l}\text { Laser: } \\
\text { Continuous } \\
\text { wave } \\
\text { LED:NR }\end{array}$ & $\begin{array}{l}\text { Laser:50 } \\
\text { mW; } 20 \mathrm{~J} / \mathrm{cm} 2 \\
\text { LED:185 } \\
\mathrm{mW} ; 46.2 \mathrm{~J} / \mathrm{cm} 2\end{array}$ & $\begin{array}{l}\text { Laser:20 J/ } \\
\text { implant } \\
\text { LED:222 J/ } \\
\text { implant }\end{array}$ & $\begin{array}{l}\text { Laser:400 } \\
\text { S } \\
\text { LED:1200s }\end{array}$ & $\begin{array}{l}\text { Immediately after } \\
\text { implant insertion } \\
\text { and 3,7,10,14 days } \\
\text { later }\end{array}$ \\
\hline Ekizer(2016) & $\begin{array}{l}\text { LED(Osseopulse }{ }^{T M} \text { Biolux Research } \\
\text { Ltd., Vancouver, Canada) }\end{array}$ & $618 \mathrm{~nm}$ & NR & $20 \mathrm{~mW} / \mathrm{cm} 2$ & NR & $1200 \mathrm{~s}$ & $\begin{array}{l}\text { Once a day during } \\
21 \text { days }\end{array}$ \\
\hline Gokmenoglu(2014) & $\begin{array}{l}\text { LED(Osseopulse } \mathrm{T}^{\mathrm{TM}} \text { AR 300, Biolux } \\
\text { Research Ltd., Vancouver, British } \\
\text { Columbia, Canada) }\end{array}$ & $626 \mathrm{~nm}$ & NR & $\begin{array}{l}185 \mathrm{~mW} .46 .2 \mathrm{~J} / \\
\mathrm{cm} 2\end{array}$ & $222 \mathrm{~J} /$ implant & $1200 s$ & $\begin{array}{l}\text { Three times per } \\
\text { week for } 3 \text { weeks }\end{array}$ \\
\hline Torkzaban(2017) & $\begin{array}{l}\text { Biolase diode laser(epic10, BIOLASE, } \\
\text { Inc., Irvine, CA, USA) }\end{array}$ & $940 \mathrm{~nm}$ & $\begin{array}{l}\text { Continuous } \\
\text { wave }\end{array}$ & $\begin{array}{l}100 \mathrm{~mW} ; 28.37 \\
\mathrm{~J} / \mathrm{cm} 2\end{array}$ & $8 \mathrm{~J} /$ implant & $80 \mathrm{~s}$ & $\begin{array}{l}\text { Repeated at 2,4, } \\
6,8,10 \text {, and } 12 \\
\text { days after imlant } \\
\text { insertion }\end{array}$ \\
\hline
\end{tabular}

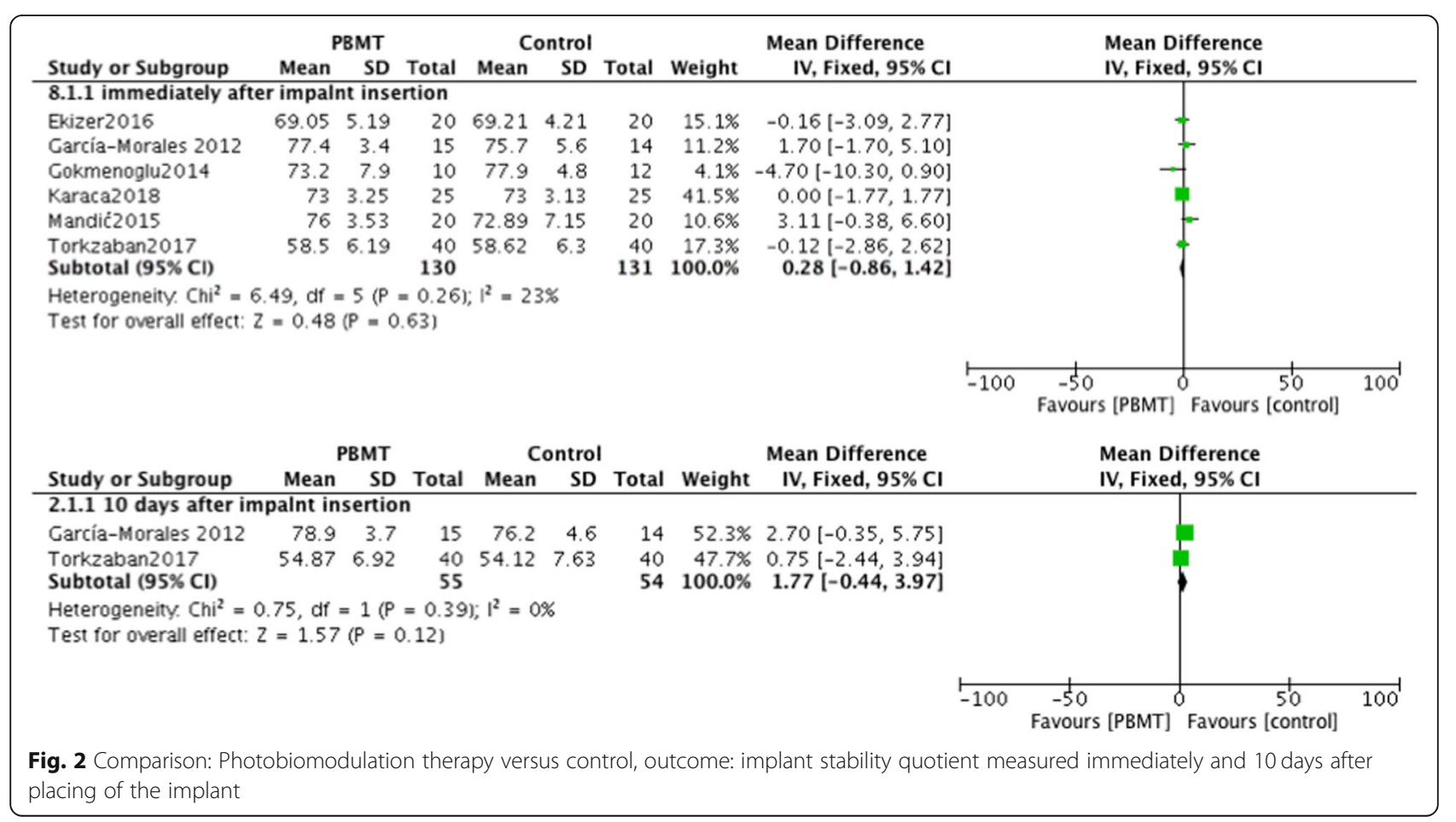




\begin{tabular}{|c|c|c|c|c|c|c|c|c|c|c|c|}
\hline \multirow[b]{2}{*}{ Study or Subgroup } & \multicolumn{3}{|c|}{ PBMT } & \multicolumn{3}{|c|}{ Control } & \multicolumn{3}{|c|}{ Mean Difference } & \multirow{2}{*}{\multicolumn{2}{|c|}{$\begin{array}{l}\text { Mean Difference } \\
\text { IV, Fixed, } 95 \% \mathrm{CI}\end{array}$}} \\
\hline & Mean & SD & Total & Mean & SD & Total & Weight & IV, Fixed, $95 \% \mathrm{CI}$ & & & \\
\hline \multicolumn{12}{|c|}{ 3.1.1 3 weeks after impalnt insertion } \\
\hline Garcia-Morales 2012 & 76.8 & 4.6 & 15 & 76.9 & 3.5 & 14 & $36.0 \%$ & $-0.10[-3.06,2.86]$ & & 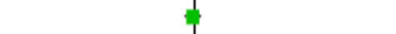 & \\
\hline Mandić2015 & 72.67 & 3.65 & 20 & 70.44 & 6.16 & 20 & $32.1 \%$ & $2.23[-0.91,5.37]$ & & 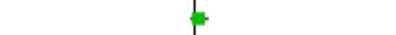 & \\
\hline Torkzaban 2017 & 54.55 & 6.75 & 40 & 53.87 & 7.59 & 40 & $31.9 \%$ & $0.68[-2.47,3.83]$ & & & \\
\hline Subtotal $(95 \% \mathrm{Cl})$ & & & 75 & & & 74 & $100.0 \%$ & $0.90[-0.88,2.67]$ & & d & \\
\hline \multicolumn{12}{|c|}{$\begin{array}{l}\text { Heterogeneity, } \mathrm{Ch}^{2}=1.15, \mathrm{df}=2(\mathrm{P}=0.56) ; \mathrm{I}^{2}=0 \% \\
\text { Test for overall effect: } \mathrm{Z}=0.99(\mathrm{P}=0.32)\end{array}$} \\
\hline & & & & & & & & & -100 & $\begin{array}{ccc}-50 & 1 & 50 \\
\text { Favours [PBMT] } & \text { Favours [control] }\end{array}$ & 100 \\
\hline \multirow[b]{2}{*}{ Study or Subgroup } & \multicolumn{2}{|c|}{ PBMT } & & \multicolumn{2}{|c|}{ Control } & & & Mean Difference & \multirow{2}{*}{\multicolumn{3}{|c|}{$\begin{array}{l}\text { Mean Difference } \\
\text { IV, Fixed, } 95 \% \mathrm{CI}\end{array}$}} \\
\hline & Mean & SD & Total & Mean & SD & Total & Weight & IV, Fixed, 95\% CI & & & \\
\hline \multicolumn{12}{|c|}{ 4.1.1 4 weeks after impalnt insertion } \\
\hline Ekizer2016 & 69.7 & 4.31 & 20 & 68.6 & 4.44 & 20 & $63.1 \%$ & $1.10[-1.61,3.81]$ & & 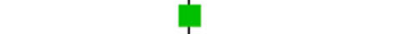 & \\
\hline Gokmenoglu2014 & 69.3 & 5.5 & 10 & 68.7 & 8.2 & 12 & $14.0 \%$ & $0.60[-5.16,6.36]$ & & I & \\
\hline $\begin{array}{l}\text { Mandić2015 } \\
\text { Subtotal }(95 \% \mathrm{Cl})\end{array}$ & 72.5 & 4.8 & $\begin{array}{l}20 \\
50\end{array}$ & 69.22 & 9.09 & $\begin{array}{l}20 \\
52\end{array}$ & $\begin{array}{r}22.9 \% \\
100.0 \%\end{array}$ & $\begin{array}{l}3.28[-1.23,7.79] \\
1.53[-0.63,3.68]\end{array}$ & & 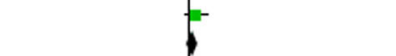 & \\
\hline \multicolumn{12}{|c|}{$\begin{array}{l}\text { Heterogeneity. } \mathrm{Chi}^{2}=0.78, \mathrm{df}=2(\mathrm{P}=0.68) ; \mathrm{I}^{2}=0 \% \\
\text { Test for overall effect: } Z=1.39(\mathrm{P}=0.16)\end{array}$} \\
\hline & & & & & & & & & -100 & $\begin{array}{ccc}-50 & 1 \\
\text { Favours [PBMT] } & \text { Favours [control] }\end{array}$ & 100 \\
\hline
\end{tabular}

participants', whereas in the other one, the methods of 'allocation concealment', 'blinding of participants', and 'blinding of personnel' were not explicitly described. In general, these methodological drawbacks may lead to bias, thereby affecting the reliability of some results. For example, selection bias can be a result of a tendency to be subjective, and contribute to false positive or negative results [18]. Imperfection of blinding techniques may exaggerate the effect, and result in false positive results [18].

Effect of PBMT on implant stability and success rate In this systematic review, 6 studies used RFA in ISQ as their measurement technique for the evaluation of

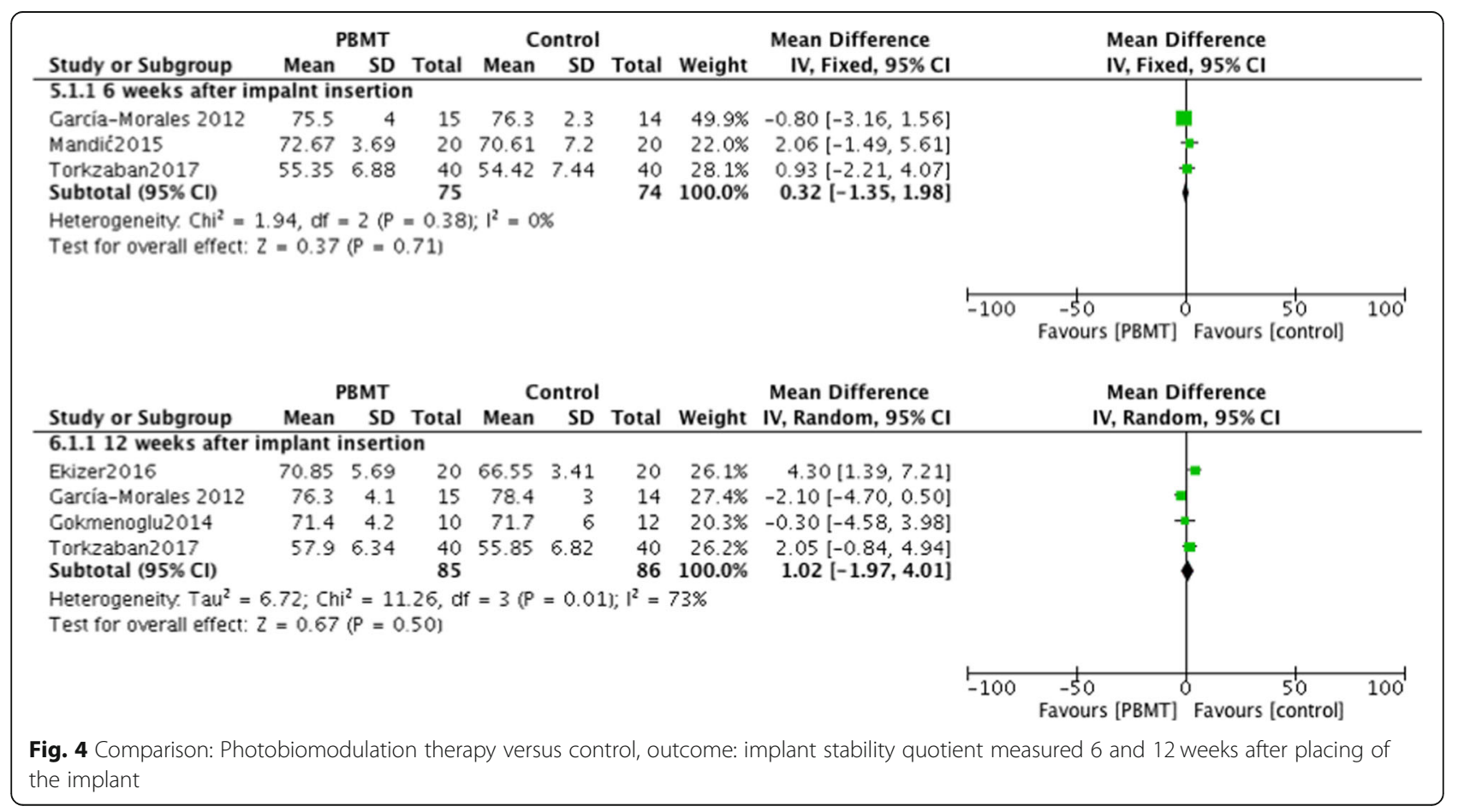




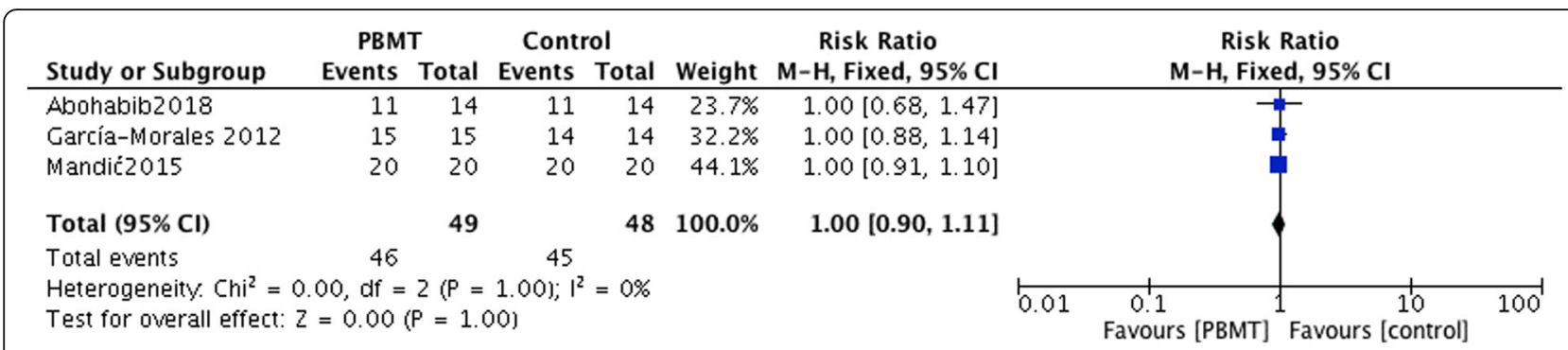

Fig. 5 Comparison: Photobiomodulation therapy versus control, outcome: success rate of implants

implant stability, for which data were pooled for meta-analysis. However, in the other 2 studies, RFA in Hertz and PTV were used respectively and were only summarized qualitatively. Objective measurements, including RFA or PTV are often used to test implant stability and osseointegration [14]. For the RFA technique, a SmartPeg is inserted on the top of the implant, then the implant resonates with the vibration of the magnetic pulse, and the frequency in Hertz would be recorded [16]. Following computer-aided data analysis, resonant frequency in Hertz is converted into an ISQ index [30]. Higher ISQ values, values varying from 1 to 100 , indicate better implant stability and better osseointegration, [2]. On the contrary, a lower PTV often indicates better implant stability with a range of -8 to 50 [1].

As shown in the meta-analysis, no significant differences were observed regarding implant success rate and implant stability (measured at days 0 and 10, and at 3, 4, 6, 12 weeks) between the PBMT group and control group. As for the other two studies, which were excluded from meta-anaysis, Memarian et al. used PTV and reported significant improvement in implant stability 3 and 4 weeks after treatment with LED or low level laser when compared with control group. Abohabib et al. used RFA in Hertz and also observed a significant increase in implant stability in the PBMT group compared with control group after 3 to 10 weeks. Although in animal studies it was established that PBMT increased bone-implant contact, and improved the production of OPG and RANKL without negative effects on bone resorption [31], existing clinical data did not provide sufficient evidence that PBMT has a positive effect on implant stability or success rate in humans. Herein, we have several hypotheses to explain the insignificant effects of PBMT on humans, which was inconsistent with the data presented in animal studies.

First of all, study design may be a factor. It is well known that a good study design is vital to obtain accurate results. However, the study design in several studies included in this review was not highly satisfactory. For example, several studies were confined to patients with a certain age range or bone condition. Thus, the data were limited and not suitable for the entire population.
Additionally, the lack of significant results may be attributed to the limited number of pooled studies and methodological defects, including inadequate countermeasures to avoid foreseeing interventions.

Secondly, the unsuitable treatment protocols of PBMT and high primary stability of implants may be a causal factor. In previous studies, it has been demonstrated that the effect of PBMT is related to the treatment protocol, including wavelength, mode, output, energy density, exposure time, and frequency of treatment [32]. Thus, unsuitable treatment protocols of PBMT may decrease the photobiomodulation efficacy on target areas, such as low energy density which does not reach the optimal therapeutic window. Moreover, most of the included studies used split-mouth design to control the experimental conditions, thus the scattering spreading energy may also affect the control sites. As indicated previously, the effect of PBMT may be masked by high primary stability of implants, which is associated with bone condition, implant surface and underized drilling technique. When the primary stability was high enough, a small change in stiffness may not be found during measuring.

Furthermore, complex human body environments would also influence the effect of PBMT. The baseline of implants in humans is often more complex compared to that in animal models. In clinical practice, patients may have dental defects caused by numerous factors, such as trauma, periodontitis or periapical periodontitis [1], following alveolar resorption, and experience severe inflammation at the site of implantation prior to surgery [31]. These complex pathological environments should be taken into consideration, because the circumstances surrounding an implant could have a significant impact on implantation outcome [32]. Additionally, the different biological nature between animals and humans may result in different biochemical reactions towards PBMT, thereby causing inconsistent treatment outcomes between the two experimental models [33-36]. It is important to note that the ossification mechanisms and function activities of tibia, femur, and human jaw bones are different. Thus, in several previously published animal studies, with implants inserted into the tibia and femur rather than the maxillary or mandibular bone, the 
placement of implants in jaw bones could not be simulated [37].

\section{Limitations of this study}

This systematic review and meta-analysis have some limitations. Firstly, only 8 studies were included, which was considerabely less than aimed for. Moreover, due to the small sample size, subjects could not be divided into corresponding groups based on age or sex [38]. Such inter-subject variations may result in clinical heterogeneity.

Secondly, baseline charateristics of bone quality in included studies varied significantly, which was reported as good in 4 studies (type 2 or 3 or reporting sufficient quantities of bone) [17-20], poor in 2 studies (type D3 or D4) [11, 22], and not disclosed in the remainder of the studies [21, 23]. Furthermore, several uncontrollable factors were present, including varying dietary habits, oral hygiene status, and awareness of oral health between patients [39]. These differences may result in inconsistent baseline parameters, which further influence collected data and analysis of the results, especially for implant stability [40]. An improved experimental design should take such factors into consideration and publicity and education in oral hygiene measures should be conduced to reduce inflammation around the implants, so as to control the baseline parameters, and acquire more reliable data [18]. Moreover, the implant material applied in all included studies was titanium. Our conclusion was limited due to the simplex impant material, which is more appropriate for titanium implants. However, since zirconia implants have gained increased attention [41], it would also be important to explore the effect of PBMT on zirconia implants.

\section{Directions for future research}

Given the limitations mentioned above, the following strategies were suggested to indicate directions for future research. Firstly, more high-quality RCTs in humans are clearly warranted to verify the data and draw rigorous conclusions, which should be strictly conducted according to the Cochrane's risk of bias criteria. Secondly, the bone conditions of all patients and PBMT parameters applied in the study should be reported in detail. Thirdly, significant concern should be taken in the effect of PBMT to different implant materials such as zirconia. At last, in order to explore the best 'therapeutic window' of PBMT, gradients for correlative PBMT parameters should be set in clinical trails.

\section{Conclusions}

Several animal studies have indicated that PBMT could facilitate hard and soft tissue regeneration, promote osseointegration, and improve implant stability, however, existing clinical studies do not support the satisfactory effect of PBMT on implant stability or the success rate in humans. Due to limitations of current study, additional high-quality human clinical trials are required to verify the data for a more convincing conclusion.

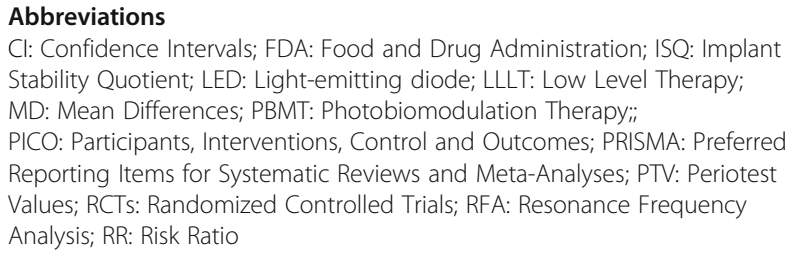

Acknowledgements

Not applicable.

Funding

There was no funding for the study.

Availability of data and materials

All data generated or analyzed during this study are included in this published article and its supplementary information files.

Authors' contributions

$A C M$ and $Y C$ were involved in the conception and planning of the study. YC, $\mathrm{CJL}$ and $\mathrm{XLC}$ conducted the literature search, carried out the data extraction and quality assessment, analyzed the data, and drafted and revised the manuscript. ACM contributed to the overall conceptualization and design of the study, served as a reviewer to resolve issues of disagreements in the selection of papers, and revised the manuscript. All authors read and approved the final manuscript.

Ethics approval and consent to participate

Not applicable.

Consent for publication

Not applicable.

\section{Competing interests}

The authors declare that they have no competing interests.

\section{Publisher's Note}

Springer Nature remains neutral with regard to jurisdictional claims in published maps and institutional affiliations.

Received: 19 January 2019 Accepted: 29 April 2019

Published online: 07 May 2019

\section{References}

1. Elieza T, Praveen A. Photobiomodulation and implants: implications for dentistry. J Periodontal Implan. 2013:43(6):262-8.

2. Prados-Frutos JC, Rodriguez-Molinero J, Prados-Privado M, Torres JH, Rojo R. Lack of clinical evidence on low-level laser therapy (LLLT) on dental titanium implant: a systematic review. Laser Med Sci. 2016;31(2):383-92.

3. Mikhail FF, Eldin M, Ibrahim T, Zekry K, Nemat AH, Nasry S. Effect of laser therapy on the Osseointegration of immediately loaded dental implants in patients under vitamin C, Omega-3 and calcium therapy. Open Access Maced J Med Sci. 2018;6(8):1468-74.

4. Matys J, Flieger R, Tenore G, Grzechleśniak K, Romeo U, Dominiak M. Er:YAG laser, piezosurgery, and surgical drill for bone decortication during orthodontic mini-implant insertion: primary stability analysis-an animal study. Laser Med Sci. 2018:33(3):489-95.

5. Mills MP, Rosen PS, Chambrone L, Greenwell H, Kao RT, Klokkevold PR, Mcallister BS, Reynolds MA, Romanos GE, Wang HL. American academy of periodontology best evidence consensus statement on the efficacy of laser therapy used alone or as an adjunct to non-surgical and surgical treatment of periodontitis and peri-implant diseases. J Periodontol. 2018;89(7):727-42. 
6. Noraian KW, Cobb CM. The efficacy of laser therapy used alone or as an adjunct to non-surgical and surgical treatment of periodontitis and periimplant diseases. J Periodontol. 2018;89(7):804-6.

7. Epstein JB, Raber-Durlacher JE, Huysmans MC, Schoordijk MCE, Cheng JE, Bensadoun RJ, Arany PR. Photobiomodulation therapy alleviates tissue fibroses associated with chronic graft-versus-host disease: two case reports and putative anti-fibrotic roles of TGF-beta. Photomed Laser Surg. 2018; 36(2):92-9.

8. Berglundh $T$, Wennström $J L$, Lindhe J. Long-term outcome of surgical treatment of peri-implantitis. A 2-11-year retrospective study. Clin Oral Implan Res. 2018;29(4):404-10.

9. Jamjoom FZ, Lee DJ. Modification of clinically short locator abutments using laser welding. J Prosthet Dent. 2018;120(1):13-6.

10. Brignardello-petersen R. Uncertainty about usefulness of low-level laser therapy for improving implant outcomes. J Am Dent Assoc. 2017;149(3):55.

11. Lau P, Bidin N, Islam S, Shukri WN, Zakaria N, Musa N, Krishnan G. Influence of gold nanoparticles on wound healing treatment in rat model: Photobiomodulation therapy. Lasers Surg Med. 2016;49(4):380-6.

12. Shahnaz A, Jamali R, Mohammadi F, Khorsand A, Moslemi N, Fekrazad R. A preliminary randomized clinical trial comparing diode laser and scalpel periosteal incision during implant surgery: impact on postoperative morbidity and implant survival. Laser Med Sci. 2017:33(1):19-25.

13. Freitas NRD, Guerrini LB, Esper LA, Sbrana MC, Dalben GDS, Soares S, Freitas $N R D$, Guerrini LB, Esper LA. Evaluation of photobiomodulation therapy associated with guided bone regeneration in critical size defects. In vivo study. J Appl Oral Sci. 2018;26:e20170244.

14. Freitas NR, Guerrini LB, Esper LA, Sbrana MC, Dalben GDS, Soares S, Almeida ALPF. Evaluation of photobiomodulation therapy associated with guided bone regeneration in critical size defects. In vivo study. J Appl Oral Sci. 2018:26:e20170244.

15. Chen Z, Zhang Y, Li J, Wang HL, Yu H. Influence of laser-microtextured surface collar on marginal bone loss and Peri-implant soft tissue response: a systematic review and meta-analysis. J Periodontol. 2017;88(7):651.

16. Momesso G, De FSB, De CS, De VL, Polo T, Hassumi JS, Garcia IJ, Faverani LP. Successful use of lower-level laser therapy in the treatment of medicationrelated osteonecrosis of the jaw. J Lasers Med Sci. 2017:8(4):201-3.

17. Muhammed SR, Rushdi SN, Abdo OS. Does the implant surgical technique affect the primary and/or secondary stability of dental implants? A systematic review. Int J Comput Dent. 2014;2014:1-17.

18. Liberati A, Altman DG, Tetzlaff J, Mulrow C, Gøtzsche PC, loannidis JP, Clarke M, Devereaux PJ, Kleijnen J, Moher D. The PRISMA statement for reporting systematic reviews and meta-analyses of studies that evaluate health care interventions: explanation and elaboration. J Clin Epidemiol. 2009;62(10):e1-34.

19. Landis JR, Koch GG. The measurement of observer agreement for categorical data. Biometrics. 1977;33(1):159-74.

20. Review Manager (RevMan) [Computer program]. Version 5, vol. 3. Copenhagen: The Nordic Cochrane Centre, The Cochrane Collaboration; 2014.

21. Higgins JPT, Green S. Cochrane handbook for systematic reviews of interventions. Chichester: Wiley-Blackwell; 2009

22. Karaca IR, Ergun G, Ozturk DN. Is low-level laser therapy and gaseous ozone application effective on osseointegration of immediately loaded implants? Photomed Laser Surg. 2018;35(10):e20170172.

23. Torkzaban P, Kasraei S, Torabi S, Farhadian M. Low-level laser therapy with $940 \mathrm{~nm}$ diode laser on stability of dental implants: a randomized controlled clinical trial. Laser Med Sci. 2018;33(2):287-93.

24. Abohabib AM, Fayed MM, Labib AH. Effects of low-intensity laser therapy on the stability of orthodontic mini-implants: a randomised controlled clinical trial. J Orthod. 2018;45(3):149-56

25. Memarian J, Ketabi M, Amini S. The effect of low-level laser $810 \mathrm{~nm}$ and light-emitting diode photobiomodulation $(626 \mathrm{~nm})$ on the stability of the implant and inflammatory markers interleukin-1 beta and prostaglandin E2, around implants. Dent Res J (Isfahan). 2018;15(4):283-8.

26. Ekizer A, Tã11/4Rker G, Uysal T, Gã1/4ray E, TaåŸDemir Z: light emitting diode mediated photobiomodulation therapy improves orthodontic tooth movement and miniscrew stability: a randomized controlled clinical trial. Lasers Surg Med 2016; 48(10):936-943.

27. Borka M, Zoran L, Aleksa M, Bojan M, Miška M, Ana D, Mi B. Influence of postoperative low-level laser therapy on the osseointegration of selftapping implants in the posterior maxilla: a 6-week split-mouth clinical study. Vojnosanit Pregl. 2015;72(3):233-40.
28. Gokmenoglu C, Ozmeric N, Erguder I, Elgun S. The effect of light-emitting diode photobiomodulation on implant stability and biochemical markers in peri-implant crevicular fluid. Photomed Laser Surg. 2014;32(3):138-45.

29. García-Morales JM, Tortamano-Neto P, Todescan FF, Andrade JCSD, Marotti J, Zezell DM. Stability of dental implants after irradiation with an 830-nm low-level laser: a double-blind randomized clinical study. Laser Med Sci. 2012;27(4):703-11.

30. Celebi H, Arpacı PU, Celik L, Akman S. The effects of laser with different parameters and crosshatching on fibroblast adhesion and proliferation to implant surfaces. Int J Oral Max Impl. 2017;32(6):1231-40.

31. Kim JR, Kim SH, Kim IR, Park BS, Kim YD. Low-level laser therapy affects osseointegration in titanium implants: resonance frequency, removal torque, and histomorphometric analysis in rabbits. J Korean Assoc Oral Maxillofac Surg. 2016;42(1):2.

32. Vanin AA, Verhagen E, Barboza SD, Costa LOP, Leal-Junior ECP. Photobiomodulation therapy for the improvement of muscular performance and reduction of muscular fatigue associated with exercise in healthy people: a systematic review and meta-analysis. Laser Med Sci. 2017; 33(1):1-34.

33. Mayer L, Gomes FV, Carlsson L, Gerhardt-Oliveira M. Histologic and resonance frequency analysis of Peri-implant bone healing after low-level laser therapy: an in vivo study. Int J Oral Max Impl. 2015;30(5):1028.

34. Kalemaj Z, Debernardi CL, Buti J. Efficacy of surgical and non-surgical interventions on accelerating orthodontic tooth movement: a systematic review. Eur J Oral Implantol. 2015;8(1):9-24.

35. Eshghpour M, Ahrari F, Najjarkar NT, Khajavi MA. Comparison of the effect of low level laser therapy with alvogyl on the management of alveolar osteitis. Med Oral Patol Oral Cir Bucal. 2015;20(3):459-68.

36. Gomes FV, Mayer L, Massotti FP, Baraldi CE, Ponzoni D, Webber JB, de Oliveira MG. Low-level laser therapy improves peri-implant bone formation: resonance frequency, electron microscopy, and stereology findings in a rabbit model. Int J Oral Max Surg. 2015;44(2):245-51.

37. Vescovi P: lasers in oral surgery and implantology. In: International conference on lasers in medicine, vol. 2016; 2016. 967007.

38. Primo BT, Da-silva RC, Grossmann E, Jr SAQM, Hernandez PAG, Jr ANS. Effect of surface roughness and low-level laser therapy on removal torque of implants placed in rat femurs. Journal of Oral Implantology. 2013;39(5):5338.

39. Khadra M, Kasem N, Lyngstadaas SP, Haanæs HR, Mustafa K. Laser therapy accelerates initial attachment and subsequent behaviour of human oral fibroblasts cultured on titanium implant material. Laser therapy accelerates initial attachment and subsequent behaviour of human oral fibroblasts cultured on titanium implant material. A scanning electron microscopic and histomorphometric analysis. Clin Oral Implants Res. 2010;16(2):168-75.

40. Pozza DH, Fregapani PW, Weber JB, de Oliveira MG, de Oliveira MA, Ribeiro NN, Jb DMS. Analgesic action of laser therapy (LLLT) in an animal model. Medicina Oral Patología Oral Y Cirugía Bucaledinglesa. 2008;13(10):E648-52.

41. Montero J, Bravo M, Guadilla Y, Portillo M, Blanco L, Rojo R, Rosales-Leal J. López-Valverde a: comparison of clinical and histologic outcomes of zirconia versus titanium implants placed in fresh sockets: a 5-month study in beagles. International Journal of Oral \& Maxillofacial Implants. 2015;30(4): $773-80$

Ready to submit your research? Choose BMC and benefit from:

- fast, convenient online submission

- thorough peer review by experienced researchers in your field

- rapid publication on acceptance

- support for research data, including large and complex data types

- gold Open Access which fosters wider collaboration and increased citations

- maximum visibility for your research: over $100 \mathrm{M}$ website views per year

At $\mathrm{BMC}$, research is always in progress.

Learn more biomedcentral.com/submission 